\title{
Reliability and efficiency upgrades of power systems operation by implementing intelligent electronic devices with synchrophasor measurement technology support
}

\author{
Alexey Mokeev ${ }^{1,2^{*}}$ \\ ${ }^{1}$ Northern (Arctic) Federal University, Electroenergetics and Electrotechnics Department, Severnaya Dvina emb.17, Arkhangelsk, Russia \\ ${ }^{2}$ Engineering Centre "Energoservice", Kotlasskaya st. 26, Arkhangelsk, Russia
}

\begin{abstract}
This paper reviews issues of reliability and efficiency upgrades of power systems functions by means of a widespread implementation of intelligent electronic devices (IED) in various purposes supporting synchrophasor measurement technology. Thus, such issues as IED's operational analysis in the conditions of electromagnetic and electromechanical transient processes and synthesis of digital filters that improve static and dynamic responses of these devices play an important role in their development.
\end{abstract}

\section{Introduction}

Successful implementation of automated WAMS by applying special PMUs devices predetermined the potential use of synchrophasor measurement technology for solving other important tasks related to reliability and efficiency upgrades of power systems operation [1-4].

The use of IEDs with synchrophasor measurement technology support allows significant improving of power systems state estimation and creating new generation devices of relay protection and automatics [2, 5]. Such advanced areas of these IEDs application are DCS substations, WACS, WAPS, WAMPACS and SmartGrid. The need for fast synchronized measurements of power system condition parameters is also associated with the wide use of electrical network and substations automatic control systems in SmartGrid by means of various regulators, including reactive power compensation devices, network parameters control devices, etc.

About one hundred power system condition parameters for fundamental harmonic can be calculated on the basis of measurements of current and voltage synchrophasors at any level of control. In addition, parameters of the power system itself can be calculated as well. Various types of emergency and automatic control, relay protection devices of new generation can be also implemented on the basis of the information coming from different IEDs.

There are higher requirements specified to IEDs used in WAPS according to main performance criteria for signal processing (speed, accuracy of signal processing, synchronism of measurements and etc.) in terms of intense electromagnetic and electromechanical transient processes. It should be noted that an existing IEEE C37.118.1 standard is focused first of all on devices used in WAMS, and does not reflect a number of specific requirements to IEDs for WAPS and WAMPACS primarily related to the need for ensuring the reliability of measurements and the required quality of nonstationary input signal processing, providing a wider range of current and voltage phasor measurements, as well as the need for a higher data rate.

The IEEE C37.118.1 standard provides for PMUs testing, corresponding to the stationary and transient conditions of the power system [6]. With an expansion of the scope for PMUs application, it is necessary to increase the number of tests corresponding to electromagnetic and electromechanical transient processes. Moreover, mathematical modelling together with testing should be more actively used as a rather simple way of IEDs functional analysis in various conditions of power system operation. At the same time, the analysis of PMUs functioning in the conditions of transients processes listed above is associated with a large amount of computation. The report examines effective methods for PMUs functional analysis during electromagnetic and electromechanical transient processes, as well as methods of express analysis.

An expansion of PMUs application is directly related to an improvement of signal processing algorithms for better IEDs quality performance such as speed and accuracy of signal processing. Along with the development of effective methods of IEDs functional analysis it requires the development of new methods for digital filters synthesis that determine static and dynamic errors of current and voltage synchrophasors measurements in most devices.

\section{PMU analysis}

According to typical signal processing algorithm provided by IEEE C37.118.1 standard [6], two main

Corresponding author: a.mokeev@narfu.ru 
operations for PMU input signal are performed here. That is signal spectrum transformation due to multiplication by a complex exponential function with a following signal filtering with the help of averaging FIRfilter. PMU functional analysis in this case comes down to a filter analysis.

According to PMUs design for ensuring correct operation under stationary conditions, as well as in the conditions of electromagnetic and electromechanical transient processes, including IEEE C37.118.1 testing, a large number of calculations are necessary. In this regard, the development of effective express methods for frequency filters analysis is an actual task for today.

Currently, similar methods of PMUs functional analysis are widely used for input sine signals on the basis of filter frequency responses. In this case, PMUs input signals are completely described by a set of complex amplitudes and imaginary frequencies of current and voltage components. Thus, requirements to the filters at PMUs design are formed on the basis of these responses. Analysis express methods for input signals corresponding to electromagnetic and electromechanical transient processes are currently under development. The article summarizes author's works related to such express methods for PMUs analysis in the conditions of electromagnetic and electromechanical transient processes [7-12]. Given methods of analysis rely on generalizing the complex amplitude method in the case of signals with constant parameters in the form of a set of damped oscillatory components and signals with variable parameters.

The methods of filter analysis below are considered for the PMU analog prototype system in order not to take into account errors of microprocessor devices associated with time and level discretization of a signal. While it should be noted that these considered methods of analysis can easily be extended to the case of discrete signals and digital filters $[8,9]$.

\subsection{Analysis for signals with constant parameters}

PMUs input signals in many practical cases can be described by a set of damped oscillatory components [8]

$$
x(t)=\operatorname{Re} \dot{\mathbf{X}}^{\mathrm{T}} e^{\mathbf{p} t}=0.5 \dot{\mathbf{X}}^{\mathrm{T}} e^{\mathrm{p} t}+0.5 \overline{\mathbf{X}}^{\mathrm{T}} e^{\overline{\mathbf{p}} t},
$$

where $\dot{\mathbf{X}}=\left[\dot{X}_{n}\right]_{N}=\left[X_{m_{n}} e^{-j \varphi_{n}}\right]_{N}-$ is the vector of input impact complex amplitude and the vector of complex frequencies is $\mathbf{p}=p_{n_{N}}=-\beta_{n}+j \omega_{n_{N}}, \overline{\mathbf{X}}, \overline{\mathbf{p}}-$ is the complex-conjugate vectors.

Unlike the traditional method of complex amplitudes, signal (1) operates with complex frequencies. Signals (1) can be presented not only by signals of the normal mode of the power system as a set of harmonics, but signals at electromagnetic transient processes, for example, a signal in a set of a sine signal and free components of transient processes (exponential and/or damped oscillatory components), and in other cases PMUs signals at some electromechanical transient processes, for example, in the case of PMU tests according to IEEE C37.118.1 for amplitude and phase modulation.

The input signal FIR-filter accounting multiplication of signal (1) by complex exponential function $2 e^{-j \omega_{0} t}$

$$
\dot{z}(t)=\dot{\mathbf{X}}^{\mathrm{T}} e^{\mathbf{p}-\mathbf{C} j \omega_{0} t}+\overline{\mathbf{X}}^{\mathrm{T}} e^{\overline{\mathbf{p}}-\mathbf{C} j \omega_{0} t}=\dot{\mathbf{Z}}^{\mathrm{T}} e^{\mathbf{p}_{1} t},
$$

where $\omega_{0}-$ nominal frequency of power system, $\mathbf{C}=1_{N}, \dot{\mathbf{Z}}=\left[\begin{array}{ll}\dot{\mathbf{X}} & \overline{\mathbf{X}}\end{array}\right], \mathbf{p}_{1}=\mathbf{p}-\mathbf{C} j \omega_{0} \quad \overline{\mathbf{p}}-\mathbf{C} j \omega_{0}$.

According to expression (2) the input signal of FIRfilter contains 2 times more components than PMU input signal (1). In this case, from one useful component of PMU input signal (1) of industrial frequency $\omega_{1}$ with complex amplitude $\dot{X}_{1}$ at the input of the filter, we have two components; the low-frequency component (constant component at $\omega_{1}=\omega_{0}$ ) and the component with frequency $\omega_{1}+\omega_{0}\left(2 \omega_{0}\right.$ at $\left.\omega_{1}=\omega_{0}\right)$. The first component should pass through the FIR-filter without any distortions, and another one should be suppressed.

It should be noted that under the conditions of stationary mode of the power system and electromagnetic transient processes such PMU input signal (1) contains one useful component of fundamental harmonic $\left(\dot{X}_{1}, \omega_{1}\right)$. As for modulated PMU input signals (amplitude and phase modulation according to IEEE C37.118.1 tests the useful signal is described as a set of complex amplitudes and imaginary frequencies. Further we are going to consider the case with one useful component of input signal (1).

As for express analysis of PMU filters at input signals (1) there are several methods [8] developed on the basis of generalizing the complex amplitude method and corresponding extended frequency responses in coordinates of the complex frequency. Let's study the analysis method based on the use of the time-dependent transfer function

$$
K(p, t)=\int_{0}^{t} g(\tau) e^{-p \tau} d \tau,
$$

where $g(t)$ - impulse function of the filter.

The output signal of the filter can be fully described by a set of time-dependent complex amplitudes

$$
\dot{\mathbf{Y}}(t)=\dot{\mathbf{Z}} \circ K \mathbf{p}_{1}, t \text {, }
$$

where $\circ-$ operation of vector multiplication.

Each time-dependent complex amplitude of vector $\dot{\mathbf{Y}}(t)$ contains information both on a forced and free component of the own transition process in the filter. At $t \rightarrow \infty$ the expression is converted to the form

$$
\dot{\mathbf{Y}}=\dot{\mathbf{Z}} \circ K \mathbf{p}_{1}
$$

and instead of a set of time-dependent complex amplitudes $\dot{\mathbf{Y}}(t)$, we get a set of complex amplitudes $\dot{\mathbf{Y}}$ corresponding to those forced components of filter reaction.

The output signal of the FIR-filter, which is synchrophasor estimation, is determined as follows

$$
\hat{X}_{1} t=\operatorname{Re} \dot{\mathbf{Y}}(t)^{\mathrm{T}} e^{\mathbf{p}_{1} t} .
$$

It should be noted that this method of analysis can be extended to cases where PMU input signal is described 
by a set of signals in the form of finite damped oscillatory components $[8,9]$.

On the basis of above expressions (4) and (5), we obtain a simple analytic method for filter analysis using only algebraic operations on complex amplitudes and values of extended frequency responses of the filter at complex frequencies of the input signal. Thus, the express analysis of filters is carried out on the basis of extended frequency responses $[7,8]$.

Express analysis, if necessary to take into account the quality of the own transient processes in the filter, is performed on the basis of the time-and-frequency approach based on the construction of frequency responses $K(p, t)$ in coordinates of the complex frequency. In most simply cases, it is preferable to use express analysis on the basis of frequency responses $K(p)$ when using spectral representations of the Laplace transform.

In the first case we are dealing with 4D MFR and FFR of the filter, and in the second case with 3D MFR and FFR. Magnitude frequency and phase-frequency function of the filter in the first case will be functions of three variables, i.e. responses must be displayed in fourdimensional space. For practical visualization of frequency responses, we can use an approach based on a three-dimensional frequency responses with a fixed value of the real or imaginary part of the complex frequency.

Let's consider the express analysis of the simplest averaging FIR-filter with a rectangular time window length $T_{0}=20 \mathrm{~ms}$ with an input signal as a set of a sine component of industrial frequency and a free exponential component of the transients process in the power system. PMU input signal in this case can be described by the set of the following complex amplitudes and frequencies $\dot{\mathbf{X}}=\left[\begin{array}{ll}\dot{X}_{1} & -X_{2}\end{array}\right], \mathbf{p}=j \omega_{1}-\beta_{2}$. Let's set the ranges of parameter variations of the useful signal and the exponential disturbance: $\omega_{1}=2 \pi 45 \div 55 \mathrm{rad} / \mathrm{s}$, $\beta_{2}=2 \div 200 \mathrm{~s}^{-1}, X_{2 \max }=\left|\dot{X}_{1}\right|$.

The input signal of the filter is completely determined by the following set of complex frequencies and amplitudes

$$
\begin{aligned}
& \mathbf{p}_{1}=\left[\begin{array}{llll}
j \omega_{1}-\omega_{0} & j & \omega_{1}+\omega_{0} & -\beta_{2}-j \omega_{0}
\end{array}\right], \\
& \dot{\mathbf{Z}}=\left[\begin{array}{lll}
\dot{X}_{1} & \bar{X}_{1} & -2 X_{2}
\end{array}\right] \text {. }
\end{aligned}
$$

Fig. 1 shows 3D MFR of the filter when using the spectral representations of the Laplace transform.

As follows from the above dependence (5), the values of the transfer function $K(p)$ of the filter at complex frequencies of the components of the filter input signal determine the variation law of the corresponding complex amplitudes. Therefore, it is quite enough to consider two cross-sections of 3D MFR here: $p=j \omega$ (curve 1) and $p=-\beta+j \omega_{0}$ (curve 2).

These two cross-sections determine variation laws of three complex amplitudes of filter input signals: the first two complex amplitudes are determined by the crosssection $p=j \omega$, and the third complex amplitude is determined by the cross-section $p=-\beta+j \omega_{0}$. To simplify the analysis with exponential disturbance at PMU input, MFR is multiplied by function $e^{-\beta T_{0}}$. The factor $e^{-\beta T_{0}}$ at $\beta \neq 0$ takes into account the damping of the aperiodic forced components by the end of the transient process in the filter. The FIR-filter speed is determined by its length $T_{0}$ only if damped components of the disturbance are suppressed to the level of the permissible error for a time equal to the length of the filter.

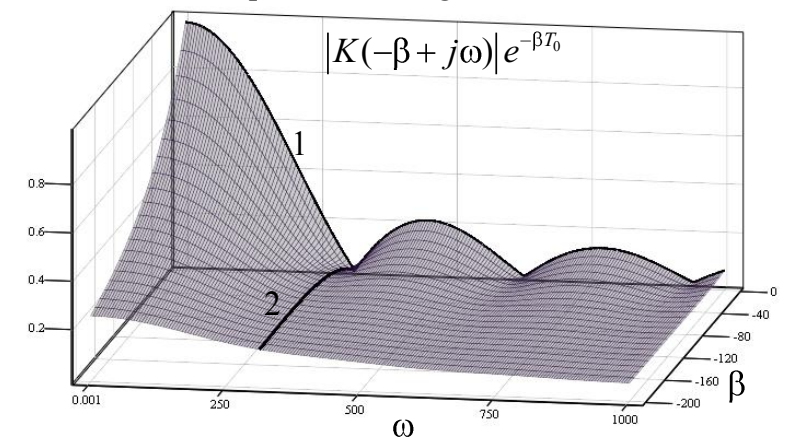

Fig. 1. 3D MFR in coordinates of complex frequency

For a more detailed analysis of filter suppressed component from the exponential disturbance we plot the function graph $2\left|K\left(-\beta+j \omega_{0}\right)\right| e^{-\beta t}$ (Fig. 2).

As follows from the graphs (Fig.1-Fig.3), the analysed filter has large errors in the estimation of the synchrophasor at the deviation of power system frequency from the nominal value, as well as has insufficient suppression of the component from exponential disturbance, especially at damping decrement $\beta_{2}=20 \div 60 \mathrm{~s}^{-1}$. So, accounting an allowable error of $5 \%$, the filter suppression time of the component from exponential disturbance is more than $50 \mathrm{~ms}$.

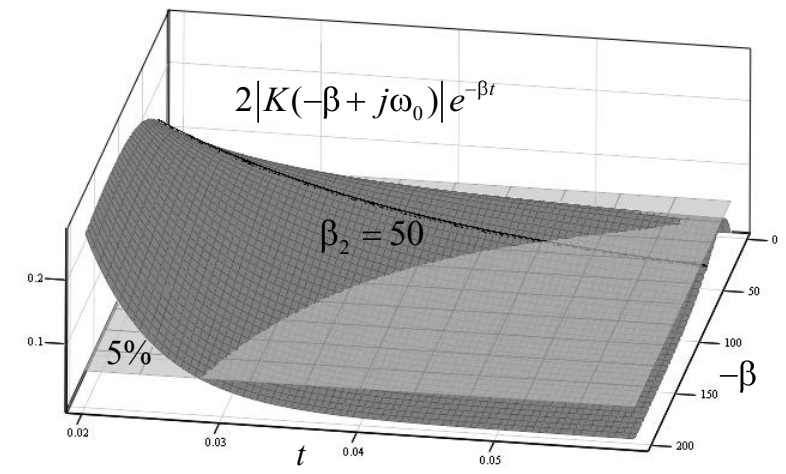

Fig.2. Exponential disturbance suppression

To analyse the filter taking into account the quality of the own transient processes in the filter, one should use extended time-and-frequency responses of the filter based on the time-dependent transfer function (3).

Fig. 3 and Fig. 4 show 3D MFR of the filter: $p=j \omega$ (Fig. 3) and $p=-\beta+j \omega_{0}$ (Fig. 4), respectively. Curves 1 and 2 in Fig. 3 correspond to the envelope of the transient process in the filter for the useful component for the synchrophasor estimation at $\omega_{1}=\omega_{0}$ and disturbance caused by multiplying PMU input signal by a complex exponential function. Curve 1 in Fig. 4 
corresponds to the transient process from the exponential component at the input of the PMU.

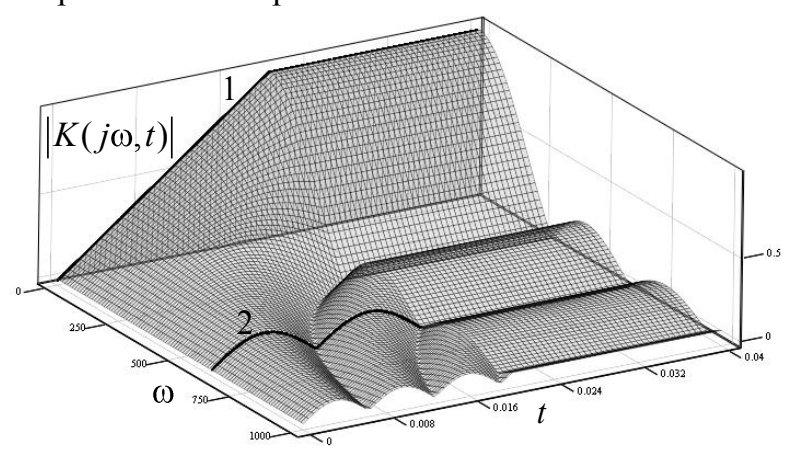

Fig. 3. Extended MFR filter (section $p=j \omega$ )

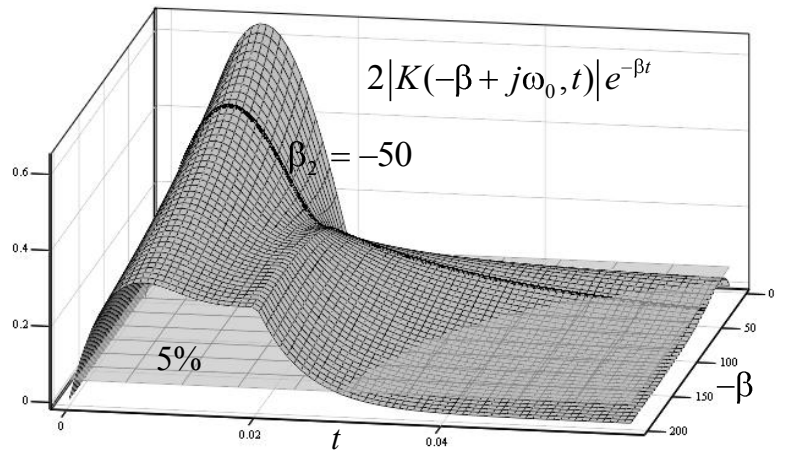

Fig.4. Extended MFR filter (section $p=-\beta+j \omega_{0}$ )

These graphs clearly demonstrate the efficiency of the proposed methods for express analysis of filters for PMU signals, which can be represented by a set of damped oscillatory components.

\subsection{Analysis for signals with variable parameters}

For the sake of simplicity, we shall restrict our analysis to the analysis of FIR-filters with only one component of PMU input signal with variable parameters, as given in IEEE C37.118.1 standard.

$$
x(t)=\operatorname{Re} \dot{X}_{1}(t) e^{j \omega_{0} t}=0.5 \dot{X}_{1}(t) e^{j \omega_{0} t}+0.5 \bar{X}_{1}(t) e^{-j \omega_{0} t} .
$$

Then the signal at the input of the FIR-filter

$$
\dot{z}(t)=\dot{X}_{1}(t)+\bar{X}_{1}(t) e^{-j 2 \omega_{0} t}=\dot{\mathbf{Z}}(t)^{\mathrm{T}} e^{\mathbf{p}_{1} t},
$$

where $\dot{\mathbf{Z}}(t)=\left[\begin{array}{lll}\dot{X}_{1} & t & \bar{X}_{1} t\end{array}\right], \mathbf{p}_{1}=\begin{array}{ll}0 & -j 2 \omega_{0}\end{array}$.

For the analysis of PMU functioning, the definitions of complex amplitudes as components of synchrophasor estimation are actual, i.e. the use of generalizing complex amplitudes and methods for filter analysis in the case of signals with variable parameters corresponding to electromechanical processes in the power system.

The determination of complex amplitudes for two components of the filter output signal can be made on the basis of the following proposed dependence [11]

$$
\dot{\mathbf{Y}}(t)=\int_{0}^{t} \dot{\mathbf{Z}} \tau \circ K^{\prime} \mathbf{p}_{1}, t-\tau d \tau,
$$

where $K^{\prime} p, t=\frac{d}{d t} K \quad p, t$
At $\dot{\mathbf{Z}} t=\dot{\mathbf{Z}}$ the expression for complex amplitudes calculation generates dependence (4).

Under certain assumptions (signal modulation depth, rate of change of frequency), complex amplitudes can be approximately estimated using the following dependence [11]

$$
\dot{\mathbf{Y}}(t) \approx \dot{\mathbf{Z}} t \circ K \quad \mathbf{p}_{1} t, t,
$$

where $\mathbf{p}_{1} t=\left[\begin{array}{lllllll}j & \omega_{1} t-\omega_{0} & j & \omega_{1} t+\omega_{0}\end{array}\right]$,

$\omega_{1}(t)=\omega_{1}+\frac{d \arg \left(\dot{X}_{1}(t)\right)}{d t}$.

The use of simplified dependence (6) for determining complex amplitudes of the output signal of the filter makes it possible to use previously discussed methods for express analysis of filters at PMU input signals with variable parameters that correspond to electromechanical processes in the power system. It is necessary to note the important advantage of the proposed methods of analysis, which is associated with the possibility of formalizing the requirements for frequency responses of filters in coordinates of the complex frequency that makes it possible to simplify the solution of the task of filters synthesis for PMU.

\section{Filters synthesis}

The method of FIR-filters synthesis [8, 9, 12] proposed by the author is based on the following basic concepts: the use of extended spectral representations of signals and filters based on the Laplace transform [7] and methods of nonlinear optimization with constraints [13]. In this case, the restrictions are imposed on $4 \mathrm{D}$ or $3 \mathrm{D}$ frequency responses of the filters.

If it is necessary to provide only the specified quality of signal processing in the filter in a steady-state condition, the constraints are imposed on 3D frequency responses. If it is necessary to additionally ensure the necessary quality of the own transient processes in the filter, then it is necessary to impose constraints on 4D frequency responses of the filters. During the synthesis of frequency filters, taking into account the nature of the input signals of the IEDs, it is advisable to use the impulse responses of filters in a set of semi-infinite (IIRfilters) or finite (FIR-filters) damped oscillatory components [8].

The efficiency of the proposed synthesis method for PMU is discussed in detail in [12]. Thus, for PMU of class $\mathrm{M}$, it was possible to double the speed compared to the filter proposed in IEEE C37.118.1 standard with the same accuracy requirements for the deviation of the frequency of the power system from the nominal value, with the same requirements for suppressing the sine component of the double frequency resulting from the operation of the spectrum shift of PMU input signal, as well as the suppression of higher harmonics and interharmonics. With a slight modernization in the signal processing algorithm for PMUs, the speed has been increased by 2 times more.

Let's consider the synthesis of FIR-filters for PMU of class $\mathrm{P}$, as well as multifunctional IEDs, performing 
the functions of PMU of class $\mathrm{P}$ and devices of relay protection and automatics.

Fig. 5 and 6 show the impulse and magnitude frequency responses of FIR-filters for the following cases, respectively: newly synthesized filters (curves 3 , $4,5,6)$ recommended by IEEE C37.118.1 for PMU of class $\mathrm{P}$ (curve 1), widely used in microprocessor-based relay protection and automatics devices (curve 2).

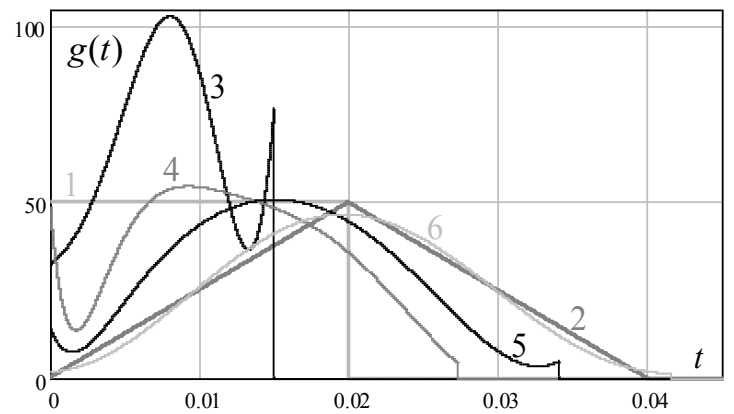

Fig. 5.Impulse responses filters

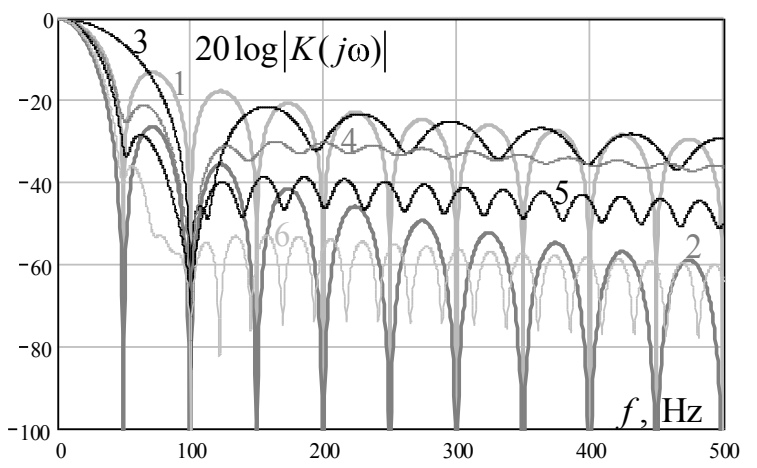

Fig. 6. MFR filters (section $p=j \omega$ )

All synthesized filters, except for the first one (curve 3 in Fig. 5 and Fig. 6), are developed for PMU modernization algorithm [12]. Here, the requirements for the permissible range of frequency variation are substantially tightened: $\pm 5 \mathrm{~Hz}$ instead of $\pm 2 \mathrm{~Hz}$ in accordance with IEEE C37.118.1 standard.

The first of the synthesized filters (curve 3) has an accuracy class 0.5 and the shortest length of filter impulse response. This filter can be used only in devices for synchronized phasor measurements of voltage in a homogeneous electrical network, when exponential components are practically absent in a voltage.

Three other synthesized filters provide suppression of the component from the exponential disturbance at the input of the device, respectively, by 20,50 and 100 times. The last filter (curve 6 in Fig. 5 and Fig. 6) provides high accuracy of measurements (accuracy class 0.2 ).

Fig. 7 shows MFR of the fourth synthesized filter (curve 5 in Fig. 5 and Fig. 6) in cross-section $p=-\beta+j \omega_{0}$, where this filter provides a given level of the suppressed component from exponential disturbance. In view of this circumstance, these filters have better speed than the widely used in relay protection averaging FIR-filter with rectangular impulse response of $20 \mathrm{~ms}$.
We would like to note the following peculiarity of the impulse responses of synthesized filters, consisting in the asymmetrical form of these responses. At the same time, we would like to draw your attention to the fact that the impulse responses inverted with respect to the axis of ordinates will have exactly the same traditional MFR as the original ones. But such two filters with mirrorsymmetrical finite impulse responses will have different properties for suppressed exponential and damped oscillatory components. This indicates the constraints of usual frequency responses based on the Fourier transform and the appropriateness of using spectral representations on the basis of the Laplace transform.

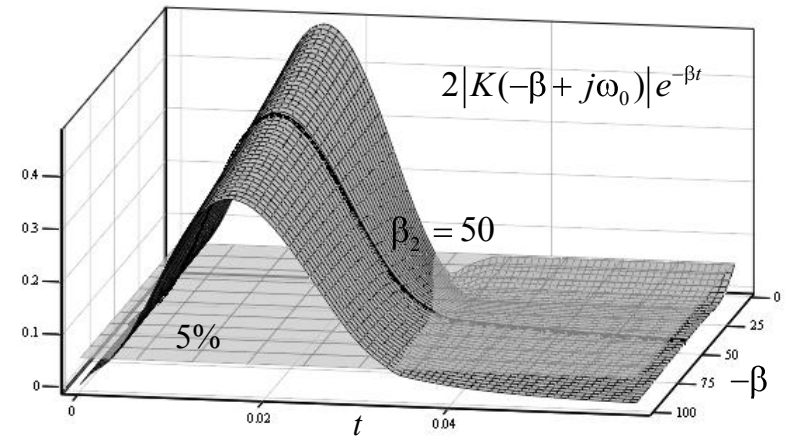

Fig. 7. MFR filter (section $p=-\beta+j \omega_{0}$ )

The conversion of analog filter prototypes to digital filters is performed using known approximation methods. Direct realization of synthesis for digital filters is also possible $[8,9]$.

\section{Implementation}

The specialists of "Engineering Centre "Energoservice" LLC developed a number of intelligent electronic devices supporting synchrophasor measurement technology, in which frequency filters synthesized using the synthesis method discussed above were implemented $[14,15]$.

Thus, the multifunctionaldeviceENIP2-PMU has been developed for use in WAMS. ENIP2-2-PMU devices can be configured for phasor measurements under class P or M. Optionally, ENIP2-2-PMU contains inboard GPS-GLONASS receiver device for time sync, as well as TFT LCD touch screen and inboard phasor data concentrator. The presence of a full set of options allows to install one PMU on the site and reduce the requirements for data transfer channels with a minimal cost.

It should be noted that ENIP2-PMU devices have very small overall dimensions in comparison with other PMUs. At present, preparations for the serial production of the multifunctional intelligent ESM device, the dimensions and cost of which are comparable with those of the multifunction measuring transducers, are being completed [15]. This makes it possible to apply them widely in the future both in SCADA and in WACS.

For use in WAMPACS and in the structure of digital substations, a multifunctional merging unit with ENMU process bus (Fig. 8) has been developed. The main 
purpose of ENMU is stand-alone merging unit (SAMU). ENMUs are connected to the relay and measuring windings of electromagnetic current transformers, as well as to measuring voltage transformers, and provide the generation of sampled values of currents and voltages in accordance with IEC 61850-9-2.

In addition, the ENMU device along with the implementation of SAMU functions performs the functions of several intelligent electronic devices, including a multifunctional measuring transducer, a synchronized phasor measurements unit, an oscillographic fault data recorder and a phasor data concentrator [15]. Phasor measurements can be considered as an addition or as an alternative to sampled values, particularly for relay protection and automatics devices, especially at implementing longitudinal differential protection and differentiation busbar protection.
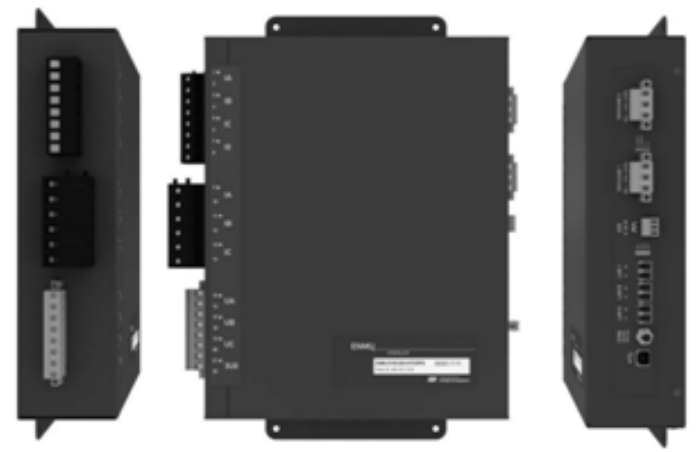

Fig. 8. Stand-Alone merging unit ENMU

The ENMU merging unit contains three optical 100BASE-FX Ethernet ports (two main and one service) and ENMU has the following protocols: IEC 61850-92LE for transmitting sampled current and voltage values (80 and 256 samples per cycle), IEEE C37. 118.2 for transmission of synchronized current and voltage phasor values, frequency and rate of change of frequency, IEC 60870-5-104 for transmission to SCADA data by measuring the parameters of the fundamental harmonic and taking into account higher harmonics. For reservation of data transfer channels, PRP backup protocol (IEC 62439-3) is implemented. The real-time clock is synchronized via a special optical port with signals PPS, IRIG-A or IRIG-B.

The ENMU device is constantly being improved. Currently, the work is underway to expand the functionality of the ENMU by adding discrete inputs and outputs and supporting the IEC 61850-8-1 protocols (MMS and GOOSE). It greatly enhances the ENMU's capabilities from the point of view of WAMPACS use.

The most important advantages of the current and voltage synchrophasors are associated with the possibility of calculating the parameters of the electrical network condition, as well as the parameters of the network itself, at any level of control. For the purpose of measuring the parameters of the network condition, taking into account the higher harmonics, work is underway to implement ENMU measurements of equivalent current and voltage synchrophasors taking into account the higher harmonics.

A prototype of the digital combined current and voltage sensor (DSCV) TECV.P1-10 with inboard merging unit has been developed for use at indoor switchgears 6-10 kV [14].

TECV.P1-10 is intended for joint use with devices of relay protection and automatics and measurements IEDs. DSCV contains a low-level electromagnetic current transformer with an inboard shunt (Low Power Current Transformer, LPCT), Rogowski coil and a capacitive voltage sensor (Fig. 9). The inboard merging unit is located in a limited space at the base of DSCV.

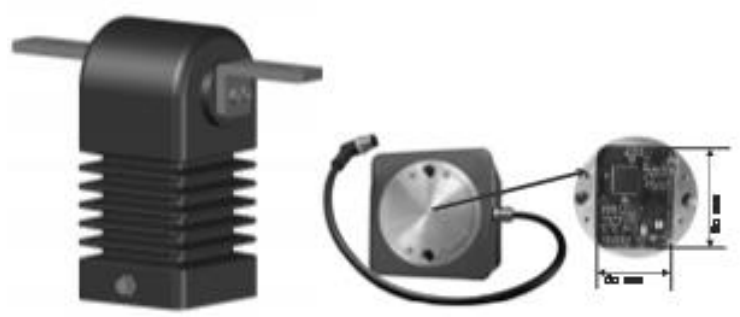

Fig. 9. Digital sensors of combined current and voltage

Measurements IEDs use digitized current data from LPCT in the range from 1 to $200 \%$ of the nominal value with accuracy class not worse than $0,2 \mathrm{~S}$. Relay protection and automatics devices use digitized current data from Rogowski coil and LPCT.

The future use of DSCV with inboard $\mathrm{MU}$ in comparison with similar sensors with analog low-level output signals is associated with better metrological performance due to the use of two current sensors, increased noise immunity, the ability to monitor DSCV and digital communication channels. The inboard AMU provides storage of data on the characteristics of current and voltage sensors and calibration coefficients[14].

DSCV is not only a source of information for the IEDs in various functional purposes, but it is also capable to perform a number of IEDs functions by implementing additional functions: telemetry, measurements of synchronized current and voltage phasors, etc. The use of synchronized current and voltage phasors measurements will significantly reduce the requirements for microcontrollers or digital signal processors of relay protection and automatics devices in $6-10 \mathrm{kV}$ switchgears.

\section{Conclusions}

The increase of the efficiency of power system control is largely determined by the quality of information coming from the information control objects. Best implementation of modern requirements for the quality of IEDs data with the support of synchrophasor measurement technology. At IED design of new generation, the task of analysing the quality of the current and voltage estimation can be effectively solved by using the above methods of express analysis of filters that determine the dynamic responses of IEDs with the support of synchrophasor measurement technology. 
The use of extended spectral representations allows us to formalize the requirements for frequency responses of filters and simplify the task of synthesizing filters for IEDs in various purposes. This, in turn, allows the implementation of more advanced IEDs supporting by synchrophasor measurement technology with extended functionality.

\section{References}

1. A.G. Phadke, J.S. Thorp, Synchronized Phasor Measurements and Their Applications (Springer, NY, 2008).

2. H.J.A. Ferrer, E.O. Schweitzer III (eds.), Modern Solutions for Protection, Control, and Monitoring of Electric Power Systems (SEL, Pullman, 2010).

3. M. Kezunovic, S. Meliopoulos et al., Application of Time-Synchronized Measurements in Power System Transmission Networks (Springer, NY, 2014).

4. Integrating Synchrophasor Technology into Power System Protection Applications (NASPI, 2016).

5. A.Z. Gamm, Y.A. Grishin, A.M. Glazunova, I.N. Kolosok, E.S. Korkina, Proceedings International Conference PowerTech (2007).

6. Standard IEEE C37.118.1-2011.

7. A.V. Mokeev, Proceedings International TICSP Workshop Spectral Methods and Multirate Signal Processing, 159-167 (TICSP, Tampere, 2007).

8. A.V. Mokeev, F.P.G. Márquez (ed.), Digital Filters, 27-52 (InTech, Rijeka, 2011).

9. A.V. Mokeev, F.P.G. Márquez and N. Zaman (ed.) Digital Filters and Signal Processing, 81-107 (InTech, Rijeka, 2013).

10. A.V. Mokeev, Proceedings IEEE International Conference SIBCON (2013).

11. A.V. Mokeev, Proceedings IEEE International Conference SIBCON (2015).

12. A.V. Mokeev, Proceedings IEEE International Conference SIBCON (2017).

13. F. Wilhelm, D. Hoffmann, Optimization - Theory and Practice (Springer, 2010).

14. A.V. Mokeev, V.N. Bovykin, L.V. Perelygin, Relay Protection and Automation, 4, 18-24 (2016).

15. A.V. Mokeev, V.N. Bovykin, E.I. Khromtsov, Relay Protection and Automation, 4, 58-63 (2015). 